\title{
Efficacy of Post-Exposure Prophylaxis in Infants Born to HBsAg Positive Mothers in Iran; Is It Authentic?
}

\author{
Zahra Ahmadinejad, ${ }^{1,}$ Zahra Abdi Liae, ${ }^{1}$ Saideh Salehizadeh, ${ }^{2}$ Sedighe Mansori, ${ }^{1}$ and Neda Alijani ${ }^{3}$ \\ ${ }^{1}$ Department of Infectious Diseases, Imam Khomeini Complex Hospital, Tehran University of Medical Sciences, Tehran, IR Iran \\ ${ }^{2}$ Faculty of Medicine, Tehran University of Medical Sciences, Tehran, IR Iran \\ ${ }^{3}$ Department of Infectious Diseases, Shariati Hospital, Tehran University of Medical Sciences, Tehran, IR Iran \\ "Corresponding author: Zahra Ahmadinejad, Department of Infectious Diseases, Imam Khomeini Complex Hospital, Tehran University of Medical Sciences, Tehran, IR Iran. Tel: \\ +98-2166581598, E-mail:ahmadiz@tums.ac.ir
}

Received 2016 March 11; Revised 2016 April 22; Accepted 2016 May 08.

\begin{abstract}
Background: Hepatitis B infection is a universal concern. This infection can lead to chronic liver disease and hepatocellular carcinoma. Neonates born to HBsAg-positive mothers are at high risk of chronic hepatitis B virus (HBV) infection, especially for HBeAgpositive mothers or neonates who have not received hepatitis B immunoglobulin (HBIg) and HBV vaccines.

Objectives: The aim of this study was to evaluate the efficacy of post-exposure prophylaxis in these infants to prevent infection.

Patients and Methods: Thirty-eight infants born to HBsAg-positive mothers between September 2006 and September 2013 were followed. The investigation evaluated whether the standard prevention protocol of neonatal HBV transmission including HBIg at birth and receiving three doses of vaccine at birth and 2 and 6 months of age was performed, followed by post-vaccination tests (evaluation of HBsAg and HBsAb titer at 9 to 18 months of age) to determine subsequent infection. HBsAb titer $\geq 10$ was considered as criterion for effectiveness of the prophylaxis procedure. The acquired data were analyzed using SPSS software (Version 18). The results are reported in descriptive tabulations.

Results: Ninety seven percent (97\%) of infants received HBIg at birth in the hospital. Generally, all of them received the first, second and third doses of vaccine at birth, 2 months, and 6 months after birth, respectively. Information for 35 mothers infected with HBV and 38 infants was available. The mean age of the mothers was 30.3 years. The results indicated that $20 \%$ of mothers were $\mathrm{HBeAg}$ positive. HBsAg was positive in one (2.6\%) infant born to an HBeAg-positive mother. Around 94\% of infants' HBsAb titers were $\geq 10$, and $5.8 \%$ were reported as non-responders.

Conclusions: The vertical transmission prevention program used in the study population in Tehran, which had an appropriate sample size, is effective. Additional doses of the vaccine can be useful in raising the effectiveness of immunoprophylaxis for infants at high risk of $\mathrm{HBV}$ infection. Also, emphasis must be set on post-vaccination testing.
\end{abstract}

Keywords: Hepatitis B Virus (HBV), HBV Vertical Transmission Prevention, HBsAg, HBeAg, Hepatitis B Immunoglobulin (HBIG)

\section{Background}

Chronic hepatitis B virus (HBV) is endemic in many areas of the world, including Asia, Africa, and the Pacific islands $(1,2)$. HBV infection is a major cause of morbidity and death throughout the world due to cirrhosis, liver failure, or liver cancer (3). Perinatal mother-to-child transmission (or perinatal vertical transmission) is the most important factor in the persistence of the HBV as endemic, and it is the common route of infection due to blood exchange during the childbirth process $(4,5)$. Depending on maternal HBV viral load and hepatitis B type e antigen (HBeAg) status and in the absence of effective immunoprophylaxis, the rates of perinatal HBV transmission are approximately $20 \%$ to $95 \%(6,7)$. Ninety percent of HBeAg-positive mothers transmit HBV infection to their offspring compared to only $10 \%-20 \%$ of HBeAg-negative mothers (8). The chance of chronic HBV infection in newborns infected with HBV perinatal transmission is $90 \%$, while risk of development of chronic HBV infections through infected adults is less than $10 \%$ (9). Twenty-four percent of adults who were infected at birth will die because of HBV-related liver disease (10).

Screening pregnant women for HBV, administering $\mathrm{HBV}$ vaccine, and administering hepatitis B immune globulin (HBIG) at birth for newborns of infected mothers are effective ways of preventing perinatal transmission that could result in markedly reduced prevalence of HBV infection in the whole population $(11,12)$. Despite the adequate administration of hepatitis B immune globulin and $\mathrm{HB}$ vaccine at birth, around 5\% to $10 \%$ of perinatal vertical transmissions of HBV could not be completely eliminated $(13,14)$. Moreover, administration of antivirals in late pregnancy for mothers with high viral loads has been shown to be an effective method of preventing perinatal transmis- 
$\operatorname{sion}(7)$.

Effectiveness of postnatal immunoprophylaxis indicated that HBV vertical transmission of infection from mothers to their newborns occurs generally during childbirth or the perinatal period rather than during pregnancy. As a result, some factors related to childbirth such as prolonged labor $(13)$, mode of delivery $(15,16)$; prematurity (17), premature rupture of membranes (18), maternalfetal hemorrhage (19), and breastfeeding might be associated with an increased risk of mother-to-child HBV transmission.

The prevalence of hepatitis B in pregnant women has been determined by the presence of hepatitis B surface antigen (HBsAg) in blood samples (20). Prevalence of hepatitis $B$ is highly variable and is dependent on region, even within a country $(21,22)$. In a study in Northern Iran (Amol), its prevalence rate among pregnant women was reported as $0.42 \%(23)$. The recommended components of perinatal HBV prevention programs also differ by region $(24,25)$. Studies in different countries have shown that the percentage of HBsAg infections has been decreased by vaccination and injection of immunoglobulin in newborns (2630). The combination of vaccination and immunoglobulin is more effective than using just one of the two (31). Failure of immunoprophylaxis could be high in mothers with positive HBeAg and high levels of HBV DNA (32). Serologic results of newborns of HBsAg-positive mothers after receiving immunoglobulin and three doses of vaccine showed that $3 \%$ of those with HBeAg mothers were HBsAg positive (33). In a study in Iran, 38.7\% of newborns with HBsAgpositive and HBeAg-positive mothers became HBsAg positive, even after receiving immunoglobulin and vaccination (34). In another study, just $45.1 \%$ of infants produced appropriate levels of antibody after receiving immunoglobulin and vaccination (35). However in a study in northern Iran (Amol) passive-active immunoprophylaxis was highly effective among high risk babies and $88.4 \%$ of children became anti-HBs positive (23). Because the results of prophylaxis efficacy studies in Iran differ from results of other studies around the world and in different regions of Iran, we were provoked to study the effectiveness of prophylaxis after exposure in infants born to positive HBsAg-positive mothers.

\section{Objectives}

This study aimed to determine the efficacy of vaccines and immunoglobulins in the prevention of hepatitis B in infants born to HBsAg-positive mothers. The results of this study verify the effectiveness of prophylaxis after exposure to prevent $\mathrm{HBV}$ transmission by the perinatal method.

\section{Patients and Methods}

In this sectional study, newborns were selected from pregnant women who were positive for HBsAg and who had been referred to Imam Khomeini hospital between September 2008 and September 2013. Thirty-five mothers were selected, of whom three had twins pregnancy, resulting in 38 newborns entering the study. None of these mothers had hepatitis D.

\subsection{Performance}

According to national and international instructions, all pregnant women must be screened for hepatitis B by measuring the level of HBs-Ag during the third trimester. Routinely, levels of HBV-DNA and HBeAg have been measured in pregnant women with hepatitis B who are referred to hepatitis clinics. In the case of high viral load, they should be treated with anti-viral drugs such as lamivudine and tenofovir. In the first 12 to 24 hours after delivery, $0.5 \mathrm{~mL}$ of hepatitis B vaccine and immunoglobulin were injected separately into gluteal muscle. All newborns in this study, at the age of 9 to 18 months, or 1 to 3 months after receiving the last of three vaccinations, were check for HBsAg and HBsAb. Newborns were divided into three groups: the first group received immunoglobulin and three complete doses of vaccine; the second group received incomplete immunoprophylaxis with immunoglobulin and fewer than three doses of vaccine, or just vaccination; the third group received no immunoprophylaxis.

In all pregnant women referred to Imam Khomeini hospital in Tehran, prophylaxis effectiveness was assessed by HBsAb. HBsAb $\geq 10$ was considered positive and HB$\mathrm{sAb}<10$ was considered negative. Results of prophylaxis were interpreted as follows: positive HBsAg was considered as a non-effective result for prophylaxis, negative HBsAg and positive HBsAb were considered as effective, both negative HBsAg and HBsAb were considered as borderline, and those newborns without post-vaccination tests were considered as an unknown group.

Effectiveness of immunoprophylaxis was evaluated according to various variables such as mothers' age, viral load level, viral treatment before or during pregnancy, and mothers' positive or negative HBeAg. Prophylaxis effectiveness was a dependent variable, while mothers' age, delivery type, newborns' age at birth, number of births, age at pregnancy, method of prophylaxis, lamivudine consumption during pregnancy, viral load, quantitative HBVDNA, and HBeAg were independent variables. Information about demographic, independent and dependent variables was gathered from questionnaires attached to 
records. In addition, more information about the effectiveness of prophylaxis was acquired from parents through phone calls.

HBsAg and HBsAb testing were done using ELISA assays with sensitivity of $100 \%$ and $98.5 \%$, respectively. HBV-DNA testing was done using polymerase chain reaction (PCR), which detects viruses at concentrations of more than 50 copies per $\mathrm{mL}$.

\subsubsection{Inclusion and Exclusion Criteria}

Mothers with positive HBsAg during pregnancy and newborns at least 9 months of age by the end of the study were entered into the study. Mothers with positive HIV or HCV were excluded from the study.

\subsection{Ethics}

Patients' information was recorded and kept confidential. No money was received from patients under the study for tests pertaining to the plan. Other issues of the declaration of Helsinki were also taken into consideration.

\subsection{Data Analysis}

Quantitative and qualitative data were analyzed and reported using SPSS software (version 18 ) as average \pm standard deviation in number and percent.

\section{Results}

4.1. Prophylaxis Effectiveness According to Independent Variables

From September 2006 to September 2013, 79 pregnant women with positive HBsAg were referred to the Hepatitis clinic of Imam Khomeini hospital complex, a 1000-bed referral educational hospital in Tehran. Forty-one women were eliminated due to incomplete data about their infants in the clinic and no availability of information over the phone. Two cases of abortion and one case of simultaneous Hepatitis $C$ were also eliminated.

At the end, 35 mothers entered the study, of whom three bore twins. Thus, 38 newborns were investigated. None of the mothers had hepatitis $D$. The average age of mothers was $30.4 \pm 6.0$ (range, 19 - 41) years. Average number of live deliveries by the mother was $2.1 \pm 1.3$ with a range of 1 to 6 deliveries. Four (11.4\%) mothers had history of one abortion each. The average gestational age of newborns at the moment of birth was $37.9 \pm 1.2 \mathrm{~s}$ (range, 36 - 41) weeks, and the average weight was $3061.3 \pm 622.3$ (range, 1800 - 4300) gr. None of the newborns had immunodeficiency disease.

After prophylaxis, HBsAg and HBsAb were assessed 17 months after birth, except for one infant (with unknown effectiveness) in whom HBsAg testing was not performed. In the other 37 infants, HBsAg tests were done, and 1 test was HBsAg-positive and 37 tests were HBsAg-negative. Of the 36 infants with positive HBsAb test, 1 was HBsAb negative and 35 were HBsAb-positive. In other words, of the 38 infants included in this study, prophylaxis was effective in 35 (HBsAg = negative, $\mathrm{HBsAb}>10$ ); in one infant it was noneffective (HBsAg = positive); in one infant it was borderline ( HBsAg = negative, HBsAb < 10); and in one infant effectiveness was unknown. The effectiveness of prophylaxis in relation to the independent variables is presented in Table 1. These results show that there was premature rupture of membranes among mothers of 4 (10.5\%) of the newborns, but prophylaxis in all of these infants was effective.

Of the 38 mothers whose newborns showed effective immunization, 17 (48.6\%) had education levels under high school diploma, 15 (40.9\%) had high school diploma or equivalent education, and 3 (8.6\%) were university graduates. Mothers of 3 newborns with borderline, non-effective, and unknown immunization had an education level under high school diploma.

Of the 35 newborns who showed effective immunization, 15 (42.9\%) were born per vaginal and 20 (57.1\%) by cesarean section. The newborn with borderline immunization was also born by cesarean section, and those with non-effective and borderline immunization were born per vagina.

History of hepatitis among family members of newborns showed that the grandmothers of 3 (7.9\%) newborns with effective immunization were HBsAg positive. Also, the fathers of 3 newborns, including 2 (66.7\%) effective and 1 (33.3\%) unknown, were positive for HBsAg. The grandmothers and fathers of the 2 newborns with hepatitis and the newborn with borderline effectiveness were not HBsAg positive. Four (10.5\%) newborns with effective immunization had other family members, including a grandfather, maternal uncle or aunts, who were HBsAg positive. Moreover, 2 newborns who showed effective prophylaxis had family members who may have had hepatitis B. Among 21 mothers with more than 1 child, 4 (19\%) mothers had other children with hepatitis B. Three (15.8\%) of the newborns with effective prophylaxis had siblings with hepatitis $B$, while there were no siblings with hepatitis B of the other 16 (84.2\%) newborns with effective prophylaxis. Also, there were siblings with hepatitis $B$ of the newborn with borderline effectiveness, and no siblings with hepatitis B of the newborn with unknown effectiveness. In addition, the newborn with hepatitis B had no siblings. 
Table 1. Prophylaxis Effectiveness in Relation to Mothers' Condition and Independent Variables ${ }^{\mathrm{a}}$

\begin{tabular}{|c|c|c|c|c|c|}
\hline Mother's Condition & Effective & Borderline & Non-Effective & Unknown & Total \\
\hline \multicolumn{6}{|l|}{ Education } \\
\hline < Diploma & $17(48.6)$ & $1(100)$ & & & $20(52.6)$ \\
\hline Diploma and associate degree & $15(42.9)$ & & & & $15(29.5)$ \\
\hline > Undergraduate & $3(8.6)$ & & & & $3(7.9)$ \\
\hline \multicolumn{6}{|l|}{ Delivery type } \\
\hline Vaginal & $15(42.9)$ & & $1(100)$ & $1(100)$ & $17(44.7)$ \\
\hline Cesarean & $20(57.1)$ & $1(100)$ & & & $21(55.3)$ \\
\hline \multicolumn{6}{|l|}{ Premature rupture of membranes } \\
\hline Positive & $4(11.4)$ & & & & $4(10.5)$ \\
\hline Negative & $31(88.6)$ & $1(100)$ & $1(100)$ & $1(100)$ & $34(89.5)$ \\
\hline \multicolumn{6}{|l|}{ Hepatitis B of the mother } \\
\hline Positive & $3(8.6)$ & & & & $3(7.9)$ \\
\hline Negative & $32(91.4)$ & $1(100)$ & $1(100)$ & $1(100)$ & $35(92.1)$ \\
\hline \multicolumn{6}{|l|}{ Hepatitis B of spouse } \\
\hline Positive & $2(5.7)$ & & & $1(100)$ & $3(7.9)$ \\
\hline Negative & $32(91.4)$ & $1(100)$ & $1(100)$ & & $34(89.5)$ \\
\hline Suspicious & $1(2.9)$ & & & & $1(2.6)$ \\
\hline \multicolumn{6}{|l|}{ Hepatitis B of other family members } \\
\hline Positive & $2(11.4)$ & & $1(100)$ & & $3(10.5)$ \\
\hline Negative & $32(82.9)$ & $1(100)$ & & $1(100)$ & $34(84.2)$ \\
\hline Suspicious & $1(5.7)$ & & & & $1(5.3)$ \\
\hline \multicolumn{6}{|l|}{ Hepatitis B of sibling } \\
\hline Positive & $3(15.8)$ & & & $1(100)$ & $4(19)$ \\
\hline Negative & $16(84.2)$ & $1(100)$ & & & $17(81)$ \\
\hline
\end{tabular}

${ }^{\mathrm{a}}$ Values are expressed as No.(\%).

4.2. Prophylaxis Effectiveness According to Mothers' Hepatitis $B$ Markers, Diagnosis Time, and Treatment

Effectiveness of prophylaxis related to diagnosis and treatment of mothers' hepatitis during pregnancy is presented in Table 2. HBV-DNA in 28 (84.8\%) mothers was positive, while in $5(15.2 \%)$ mothers it was negative, and in 2 mothers it was not recorded. Twenty-five (89.3\%) newborns of those mothers with positive HBV-DNA showed effective prophylaxis, while newborns with non-effective, borderline, and unknown effectiveness were from mothers with positive HBV-DNA. Average amount of HBV-DNA was $6,299,759.6 \pm 13,490,001.7$ IU with a maximum of $5,582,857.14$ IU and minimum of 2.5 IU. Average HBV-DNA in mothers whose newborns showed effective prophylaxis was 4,274,906.4 $\pm 12,316,683.8$ IU. Level of viral load of mothers with unknown, borderline, and non-effective prophylaxis in their newborns was $137,569,1,482$, and 40,731,263.8 IU, respectively.

Results of PCR in 34 mothers were available: in 10 (29.4\%) mothers PCR was performed in the first trimester, in 9 (26.5\%) PCR was performed in the second trimester, and in 15 (44.1\%) it was performed in the third trimester. In the mother of the newborn with hepatitis, the PCR test was done in the third trimester. Of the 38 newborns in the study, hepatitis B in 9 (23.7\%) mothers was diagnosed in the first trimester, in 12 (31.6\%) it was diagnosed in the second trimester, and in 9 (23.7\%) it was diagnosed in the third trimester, and 8 (21.1\%) of the mothers had it before pregnancy. In the mothers of one newborn with hepatitis and one with unknown effectiveness, hepatitis was diagnosed in the third trimester. Also, the hepatitis treatment of mothers was considered in this study. Eight (21.1\%) 
Table 2. Newborns' Prophylaxis Effectiveness According to Mother's Hepatitis B Status ${ }^{\mathrm{a}}$

\begin{tabular}{|c|c|c|c|c|c|}
\hline Status & Effective & Borderline & Non-Effective & Unknown & Total \\
\hline \multicolumn{6}{|c|}{ Diagnosis of hepatitis B in pregnancy } \\
\hline Before pregnancy & $9(25.7)$ & & & & $9(23.7)$ \\
\hline First trimester & $11(31.4)$ & $1(100)$ & & & $12(31.6)$ \\
\hline Second trimester & $9(25.7)$ & & & & $9(23.7)$ \\
\hline Third trimester & $6(17.1)$ & & $1(100)$ & $1(100)$ & $8(21.1)$ \\
\hline \multicolumn{6}{|l|}{ PCR implementation time } \\
\hline First trimester & $10(32.3)$ & & & & $10(29.4)$ \\
\hline Second trimester & $8(25.8)$ & $1(100)$ & & & $9(26.5)$ \\
\hline Third trimester & $13(41.9)$ & & $1(100)$ & $1(100)$ & $15(44.1)$ \\
\hline \multicolumn{6}{|l|}{ DNA PCR } \\
\hline Positive & $25(83.3)$ & $1(100)$ & $1(100)$ & $1(100)$ & $28(84.8)$ \\
\hline Negative & $5(16.7)$ & & & & $5(15.2)$ \\
\hline \multicolumn{6}{|l|}{ Anti-Viral drug } \\
\hline Yes & $6(17.1)$ & & $1(100)$ & $1(100)$ & $8(21.1)$ \\
\hline No & $29(82.9)$ & $1(100)$ & & & $30(78.9)$ \\
\hline \multicolumn{6}{|c|}{ Beginning time of anti-viral drugs } \\
\hline First trimester & $1(16.7)$ & & & & $1(12.5)$ \\
\hline Second trimester & $1(16.7)$ & & & & $1(12.5)$ \\
\hline Third trimester & $4(66.6)$ & & $1(100)$ & $1(100)$ & $6(75)$ \\
\hline
\end{tabular}

${ }^{\mathrm{a}}$ Values are expressed as No.(\%).

of the mothers were treated with anti-viral drugs during pregnancy. In one (12.5\%) mother, treatment was started during the first trimester. In one (12.5\%), treatment was started during the second trimester, and in 6 (75\%), treatment was started during the third trimester. Mothers of the newborn with hepatitis and with unknown effectiveness started treatment in the third trimester. The mother of the newborn with borderline effectiveness had not received anti-viral treatment.

Serologic tests of mothers are presented in Table 3. From 30 mothers with available HBeAg tests, 24 (80\%) were negative and $6(20 \%)$ were positive. The mother of the one newborn with hepatitis, and the mother of the infant with unknown prophylaxis effectiveness were HBeAg positive. Among newborns with effective prophylaxis, 4 (12.9\%) mothers were BeAg positive and 27 (87.1\%) mothers were HBeAg negative. Also, of 29 available HBeAb tests, 21 (72.4\%) were positive and 8 (27.6\%) were negative, and of these 29 , 28 had newborns with effective prophylaxis. No information on the HBeAb test of the mother of neonate with hepatitis was available. Sixteen HBcAb tests were available, of which 14 (87.5\%) were positive and 2 (12.5\%) were negative.
It is noticeable that 15 of these newborns showed effective prophylaxis. No information was available from mother of neonate with hepatitis. Furthermore, 15 HBsAb tests were accessible, of which 1 (6.7\%) case was positive and 14 (93.3\%) were negative. Thirteen of them had newborns who showed effective prophylaxis, and 12 (92.3\%) of these mothers were negative for HBsAb. No information was available for the HBsAb test of the mother of neonate with hepatitis.

\subsection{Prophylaxis Effectiveness According to Newborn Condition}

Table 4 presented, 11 (28.9\%) newborns were preterm, $26(68.4 \%)$ were at term, and $1(2.7 \%)$ newborn was postterm statue. All of the pre-terms, the post-term, and 23 of the term newborns showed effective prophylaxis. The newborn with hepatitis B and those with borderline and unknown effectiveness were born at term. Eight (21.1\%) of the newborns had low birth weight (LBW), lower than $2500 \mathrm{gr}$, and 30 (78.9\%) weighed more than 2500 gr. Neither the newborn with hepatitis B nor the one with unknown prophylaxis effectiveness was categorized in the LBW group. From 34 newborns with effective prophylaxis, 7 (20\%) were LBW and 28 (80\%) weighed more than 2500 gr. Moreover, 31 (81.6\%) of the newborns were breastfed and 7 (18.4\%) were 
Table 3. Results of Hepatitis Serological Tests in the Mothers Under Study ${ }^{a}$

\begin{tabular}{|c|c|c|}
\hline Serologic Test & Positive & Negative \\
\hline HBe-Ag & $6(20)$ & $24(80)$ \\
\hline HBe-Ab & $21(72.4)$ & $8(27.6)$ \\
\hline HBc-Ab & $14(87.5)$ & $2(12.5)$ \\
\hline HBs-Ab & $1(6.7)$ & $14(93.3)$ \\
\hline
\end{tabular}

${ }^{\mathrm{a}}$ Values are expressed as No.(\%).

fed with formula. The one with hepatitis was breastfed. Also, the prophylaxis' performance was incomplete in just one (2.6\%) newborn; nonetheless, the result was effective.

\section{Discussion}

Immunization of infants born to mothers with HBsAg is the best way to prevent HBV vertical transmission. Our results demonstrated that immunoprophylaxis with immunoglobulin and vaccination significantly decreased HBV transmission, similar to the results of Koyama et al. (26). Also, in a study by Lee et al., hepatitis B occurrence among newborns who received either vaccine or immunoglobulin or both was lower compared to those newborns who did not receive vaccine or immunoglobulin; the combination of immunoglobulin and vaccine is more effective than vaccine alone (31).

The mother of the only newborn with hepatitis B in our study was positive for HBeAg. According the studies of Wu et al., positive-HBeAg is almost indicative of replicative virus and increases the probability of HBV transmission to $66 \%$ to $93 \%$. Also, there was a significant correlation between higher load of HBV-DNA and intrauterine transmission (29). The mother of the newborn with hepatitis B in our study showed higher viral load. Thus, in order to diminish the perinatal transmission, consumption of anti-viral drugs is proposed. Also, it is better to inject immunoglobulin, along with vaccine, in newborns with HBeAg-positive mothers. It has been shown that the probability of HBV transmission decreased to $0.8 \%$ when immunoglobulin was combined with vaccine (36).

According to Evans et al., some of the newborns of HBeAg- positive mothers were infected, even though they had received immunization vaccines (27). In our study, one newborn with complete prophylaxis was infected. Despite $20 \%$ of mothers being HBeAg-positive, infection was successfully prevented in $97.3 \%$ of newborns. Our results are in agreement with the centers for disease control (CDC), which proposed one dose of immunoglobulin and regular vaccination to prevent $\mathrm{HBV}$ infection in newborns of $\mathrm{HB}-$ sAg positive mothers with an effectiveness of $85 \%$ to $95 \%$
(37). In another study, 204 newborns with HBsAg-positive mothers received immunoglobulin and three doses of vaccine, of whom six were HBeAg-positive mothers (33). Our results are compatible with these findings. Also, one newborn had received only three doses of vaccine (without immunoglobulin) and remained free of infection. This result implies that the efficacy of vaccination alone in preventing infection is 75\% (38), although the combination of immunoglobulin and vaccine is more effective (31).

\subsection{Conclusion}

The investigation of vertical transmission in the population sample in Imam Khomeini Complex hospital in Tehran showed that prevention of such transmission is possible. Immunoprophylaxis treatments were effective in preventing HBV transmission from mothers to infants. Moreover, an additional dose of vaccine could be more effective for those infants who are at higher risk of HBV infection to increase the immunoprophylaxis impact. Also, post-vaccination tests must be recommended for all infants to evaluate the effectiveness of this measure. 
Table 4. Newborns' Prophylaxis Effectiveness According to Their Status ${ }^{\mathrm{a}}$

\begin{tabular}{|c|c|c|c|c|c|}
\hline Status & Effective & Borderline & Non-effective & Unknown & Total \\
\hline \multicolumn{6}{|c|}{ Nourishment by breastfeeding } \\
\hline Yes & $29(82.9)$ & $1(100)$ & $1(100)$ & & $31(81.6)$ \\
\hline No & $6(17.1)$ & & & $1(100)$ & $7(18.4)$ \\
\hline \multicolumn{6}{|l|}{ Low birth weight } \\
\hline Yes & $7(20)$ & $1(100)$ & & & $8(21.1)$ \\
\hline No & $28(80)$ & & $1(100)$ & $1(100)$ & $30(78.9)$ \\
\hline \multicolumn{6}{|l|}{ Maturity } \\
\hline Pre-term & $11(31.4)$ & & & & $11(28.9)$ \\
\hline Term & $23(65.7)$ & $1(100)$ & $1(100)$ & $1(100)$ & $26(68.4)$ \\
\hline Post-term & $1(2.9)$ & & & & $1(2.6)$ \\
\hline \multicolumn{6}{|l|}{ Prophylaxis quality } \\
\hline Complete & $34(97.1)$ & $1(100)$ & $1(100)$ & $1(100)$ & $37(97.4)$ \\
\hline Incomplete & $1(2.9)$ & & & & $1(2.6)$ \\
\hline
\end{tabular}

${ }^{\mathrm{a}}$ Values are expressed as No. (\%).

\section{Acknowledgments}

We are thankful to our colleagues Dr. Mohsen Nasiri Toosi and Dr. Shahin Hantooshzadeh, who provided expertise and information that greatly assisted the research. We are also grateful to Dr. Bayani for assistance with the data gathering and Miss Felegary, who connected us to the mothers by phone.

\section{References}

1. Centers for Disease Control Prevention. Screening for chronic hepatitis B among Asian/Pacific Islander populations-New York City, 2005. MMWR Morb Mortal Wkly Rep. 2006;55(18):505-9. [PubMed: 16691180].

2. Gust ID. Epidemiology of hepatitis B infection in the Western Pacific and South East Asia. Gut. 1996;38 Suppl 2:S18-23. [PubMed: 8786047].

3. WHO . Hepatitis B fact sheet N 204 WHO,; 2014. Available from: http: //www.who.int/mediacentre/factsheets/fs204/en/.

4. Stevens CE, Neurath RA, Beasley RP, Szmuness W. HBeAg and antiHBe detection by radioimmunoassay: correlation with vertical transmission of hepatitis B virus in Taiwan. J Med Virol. 1979;3(3):237-41. [PubMed: 479860].

5. Edlich RF, Diallo AO, Buchanan L, Martin ML. Hepatitis B virus: a comprehensive strategy for eliminating transmission in the United States. J Long Term Eff Med Implants. 2003;13(2):117-25. [PubMed: 14510285].

6. Beasley RP, Hwang LY, Stevens CE, Lin CC, Hsieh FJ, Wang KY, et al. Efficacy of hepatitis B immune globulin for prevention of perinatal transmission of the hepatitis B virus carrier state: final report of a randomized double-blind, placebo-controlled trial. Hepatology. 1983;3(2):135-41. [PubMed: 6339349].

7. Patton H, Tran TT. Management of hepatitis B during pregnancy. Nat Rev Gastroenterol Hepatol. 2014;11(7):402-9. doi: 10.1038/nrgastro.2014.30. [PubMed: 24686270].
8. Stevens CE, Beasley RP, Tsui J, Lee WC. Vertical transmission of hepatitis B antigen in Taiwan. $N$ Engl J Med. 1975;292(15):771-4. doi: 10.1056/NEJM197504102921503. [PubMed: 1113797].

9. Edmunds WJ, Medley GF, Nokes DJ, Hall AJ, Whittle HC. The influence of age on the development of the hepatitis B carrier state. Proc Biol Sci. 1993;253(1337):197-201. doi: 10.1098/rspb.1993.0102. [PubMed: 8397416].

10. Chang MH, You SL, Chen CJ, Liu CJ, Lee CM, Lin SM, et al. Decreased incidence of hepatocellular carcinoma in hepatitis B vaccinees: a 20-year follow-up study. J Natl Cancer Inst. 2009;101(19):1348-55. doi: 10.1093/jnci/djp288. [PubMed: 19759364].

11. Wong VC, Ip HM, Reesink HW, Lelie PN, Reerink-Brongers EE, Yeung $\mathrm{CY}$, et al. Prevention of the HBsAg carrier state in newborn infants of mothers who are chronic carriers of HBsAg and HBeAg by administration of hepatitis-B vaccine and hepatitis-B immunoglobulin. Doubleblind randomised placebo-controlled study. Lancet. 1984;1(8383):9216. [PubMed: 6143868].

12. Beasley RP, Hwang LY, Lee GC, Lan CC, Roan CH, Huang FY, et al. Prevention of perinatally transmitted hepatitis $\mathrm{B}$ virus infections with hepatitis B immune globulin and hepatitis B vaccine. Lancet. 1983;2(8359):1099-102. [PubMed: 6138642].

13. Lin HH, Chang MH, Chen DS, Sung JL, Hong KH, Young YC, et al. Early predictor of the efficacy of immunoprophylaxis against perinatal hepatitis B transmission: analysis of prophylaxis failure. Vaccine. 1991;9(6):457-60. [PubMed: 1832257].

14. Ngui SL, Andrews NJ, Underhill GS, Heptonstall J, Teo CG. Failed postnatal immunoprophylaxis for hepatitis B: characteristics of maternal hepatitis B virus as risk factors. Clin Infect Dis. 1998;27(1):100-6. [PubMed: 9675462].

15. Lee SD, Lo KJ, Tsai YT, Wu JC, Wu TC, Yang ZL, et al. Role of caesarean section in prevention of mother-infant transmission of hepatitis B virus. Lancet. 1988;2(8615):833-4. [PubMed: 2902274].

16. Wang J, Zhu Q, Zhang X. Effect of delivery mode on maternal-infant transmission of hepatitis B virus by immunoprophylaxis. Chin Med J (Engl). 2002;115(10):1510-2. [PubMed:12490098].

17. Mulligan MJ, Stiehm ER. Neonatal hepatitis B infection: clinical and immunologic considerations. J Perinatol. 1994;14(1):2-9. [PubMed: 8169673]. 
18. Fiore AE. Chronic maternal hepatitis B infection and premature rupture of membranes. Pediatr Infect Dis J. 2002;21(4):357-8. [PubMed: 12075775]

19. Lin HH, Kao JH, Hsu HY, Mizokami M, Hirano K, Chen DS. Least microtransfusion from mother to fetus in elective cesarean delivery. Obstet Gynecol. 1996;87(2):244-8. doi: 10.1016/0029-7844(95)00385-1. [PubMed: 8559532].

20. Salisbury D, Noakes K. Immunisation against infectious diseases (The Green Book). 2 ed. London: Department of Health; 2009. pp. 161-84.

21. Boxall E, Skidmore S, Evans C, Nightingale S. The prevalence of hepatitis $B$ and $C$ in an antenatal population of various ethnic origins. Epidemiol Infect. 1994;113(3):523-8. [PubMed: 7527780].

22. Batham A, Narula D, Toteja T, Sreenivas V, Puliyel JM. Sytematic review and meta-analysis of prevalence of hepatitis B in India. Indian Pediatr. 2007;44(9):663-74. [PubMed: 17921555].

23. Yahyapour Y, Karimi M, Molaei HR, Khoddami E, Mahmoudi M. Active-passive Immunization Effectiveness Against Hepatitis B Virus in Children Born to HBsAg Positive Mothers in Amol, North of Iran. Oman Med J. 2011;26(6):399-403. doi: 10.5001/omj.2011.103. [PubMed: 22253947].

24. Lazarus J. Global policy report on the prevention and control of viral hepatitis in WHO Member States. Geneva: World Health Organization; 2014.

25. WHO . Hepatitis B vaccines: WHO position paper-recommendations Vaccine. 2010;28(3):589-90. doi: 10.1016/j.vaccine.2009.10.110. [PubMed: 19896455].

26. Koyama T, Matsuda I, Sato S, Yoshizawa H. Prevention of perinatal hepatitis B virus transmission by combined passive-active immunoprophylaxis in Iwate, Japan (1981-1992) and epidemiological evidence for its efficacy. Hepatol Res. 2003;26(4):287-92. [PubMed: 12963428].

27. Evans AA, Cohen C, Huang P, Qian L, London WT, Block JM, et al. Prevention of perinatal hepatitis $B$ transmission in Haimen City, China: Results of a community public health initiative. Vaccine. 2015;33(26):3010-5. doi: 10.1016/j.vaccine.2015.01.054. [PubMed: 25698491].

28. Hahne S, van den Hoek A, Baayen D, van der Sande M, de Melker $\mathrm{H}$, Boot $\mathrm{H}$. Prevention of perinatal hepatitis B virus transmission in the Netherlands, 2003-2007: children of Chinese mothers are at in- creased risk of breakthrough infection. Vaccine. 2012;30(9):1715-20. doi: 10.1016/j.vaccine.2011.12.071. [PubMed: 22210140].

29. Wu JS, Hwang LY, Goodman KJ, Beasley RP. Hepatitis B vaccination in high-risk infants: 10-year follow-up. J Infect Dis. 1999;179(6):1319-25. doi: 10.1086/314768. [PubMed: 10228050]

30. Mele A, Tancredi F, Romano L, Giuseppone A, Colucci M, Sangiuolo $A$, et al. Effectiveness of hepatitis B vaccination in babies born to hepatitis B surface antigen-positive mothers in Italy. J Infect Dis. 2001;184(7):905-8. doi: 10.1086/323396. [PubMed: 11509998].

31. Lee C, Gong Y, Brok J, Boxall EH, Gluud C. Effect of hepatitis B immunisation in newborn infants of mothers positive for hepatitis B surface antigen: systematic review and meta-analysis. BMJ 2006;332(7537):328-36. doi: 10.1136/bmj.38719.435833.7C. [PubMed: 16443611].

32. Song YM, Sung J, Yang S, Choe YH, Chang YS, Park WS. Factors associated with immunoprophylaxis failure against vertical transmission of hepatitis B virus. Eur J Pediatr. 2007;166(8):813-8. doi: 10.1007/s00431-006-0327-5. [PubMed:17120036].

33. Poovorawan Y, Chongsrisawat V, Theamboonlers A, Srinivasa K, Hutagalung Y, Bock HL, et al. Long-term benefit of hepatitis B vaccination among children in Thailand with transient hepatitis B virus infection who were born to hepatitis B surface antigen-positive mothers. J Infect Dis. 2009;200(1):33-8. doi: 10.1086/599331. [PubMed:19473096].

34. Alavian SM, Behnava B. What is the reason for poor outcome of antepartum immunoprophylaxis of Hepatitis B Immunoglobulin in prevention of vertical hepatitis B transmission. Hepat Mon. 2007;7(3):163-5

35. Hassanjani-Roshan MR, Zahed-Pasha Y. Efficacy of HBIG and Vaccine in infants of HBsAg positive carrier mothers. Arch Irn Med. 2002;5(1):21-3.

36. Salman K, Priti S, Molly M, Kumar VS, Zeenat S. Hepatitis B virus infection in pregnant women and transmission to newborns. Asian Pac J Trop Dis. 2015;5(6):421-9. doi: 10.1016/s2222-1808(15)60809-x.

37. CDC . Protection against viral hepatitis. Recommendations of the Immunization Practices Advisory Committee (ACIP). MMWR Recomm Rep. 1990;39(RR-2):1-26. [PubMed: 2153904].

38. Marcdante K. J. , Kliegman RM, Jenson HB, Behrman RE. Nelson Essentials of Pediatrics. 6 ed. Philadelphia: Elsevier Health Sciences; 2010. 